\title{
Sumisión química en presuntas víctimas de violencia sexual
}

\section{Chemical Submission in Alleged Victims of Sexual Violence}

Jorge Marcelo Quintana Yánez. ${ }^{1}$, María de los Ángeles Galarza Pazmiño. ${ }^{2}$, Andrea Paola Hinojosa León. ${ }^{3}$ \& Verónica Paulina Cáceres Manzano. ${ }^{4}$

Recibido: 10-02-2019 / Revisado: 15-02-209 /Aceptado: 04-03-2019/ Publicado: 12-03-2019

\begin{abstract}
.
DOI: https://doi.org/10.32/cienciadigital.v3i1.947

In Ecuador, several crimes, especially those of a sexual nature, are associated with the use of drugs, due to their effects on the organism, which facilitate the perpetrator to consummate the unlawful behavior. The relation of the hours elapsed from the presumed criminal act to the sampling and the results of the forensic toxicology examination was established; being an observational, analytical and cross-sectional study of the cases that came to the Flagrancia-Quito unit. It was found that the most used substance was alcohol $(83.3 \%)$, the way of administration was voluntary in $60.2 \%$ and the state of consciousness during the event was stupor in $35.6 \%$. There was a statistically significant moderate association between the positive results of toxicological tests and the hours elapsed since the events (Pearson's Chi2 $=10.3$, $\mathrm{p}$ $=0.001 \mathrm{Phi}=-0.450 \mathrm{~V}$ of Cramer $=0.450$ ). Conclusion: The hours elapsed from the fact to the taking of the sample are intimately related, having a higher percentage of positivity in the results of samples that were obtained earlier, maximum up to eight hours after the crime, so it is important in cases that are suspected of aggression with the use of chemical substances do not delay the legal medical examination plus the taking of samples.
\end{abstract}

Keywords: Violation, Drugs, Alcohol, Time.

\section{Resumen.}

En Ecuador, varios delitos especialmente los de índole sexual, están asociados al uso de drogas, debido a sus efectos en el organismo, que facilitan al perpetrador consumar la conducta antijurídica. Se estableció la relación de las horas

\footnotetext{
${ }^{1}$ Medico Perito Egresado del Posgrado de Medicina Forense, marcelin90@ hotmail.com

${ }^{2}$ Medico Perito Egresado del Posgrado de Medicina Forense, angelesgalarzapazmino@gmail.com ${ }^{3}$ Médico Cirujano Residente Asistencial Hospital General Latacunga,andreispao@hotmail.com ${ }^{4}$ Universidad Nacional de Chimborazo, Docente Facultad de Ciencias de la Salud, vcaceres@unach.edu.ec
} 
transcurridas desde el presunto hecho delictivo hasta la toma de muestras y los resultados el examen de toxicología forense; siendo un estudio de carácter observacional, analítico y transversal de los casos que acudieron a la unidad de Flagrancia - Quito. Se encontró que la sustancia más usada fue el alcohol (83.3\%), la manera de administración fue voluntaria en el $60.2 \%$ y el estado de conciencia durante el hecho fue el estupor en el 35,6\%. Existió una asociación moderada estadísticamente significativa entre los resultados positivos de exámenes toxicológicos y las horas transcurridas desde los hechos (Chi2 de Pearson=10.3; $\mathrm{p}=$ 0,001 Phi $=-0.450 \mathrm{~V}$ de Cramer $=0.450)$. Conclusión: Las horas transcurridas desde el hecho hasta la toma de la muestra están íntimamente relacionadas, teniendo un mayor porcentaje de positividad en los resultados de muestras que se obtuvieron más tempranamente, máximo hasta ocho horas posteriores al hecho delictivo, por lo que es importante en los casos que se sospeche de una agresión con uso de sustancias químicas no se demore el examen médico legal más la toma de muestras.

Palabras claves: Violación, Drogas, Alcohol, Tiempo.

\section{INTRODUCCIÓN}

Los delitos en contra de la integridad sexual constituyen un problema en nuestro país, existe además una cifra negra, ya que muchos casos no son denunciados y prácticamente es imposible mesurar la real magnitud del problema. El empleo de sustancias toxicas facilitadoras de conductas antijurídicas, especialmente en los de carácter sexual, plantea retos adicionales a la hora del diagnóstico, por la cuantiosa sintomatología que presenta la víctima, en especial la amnesia (1), por esta razón se incrementa el intervalo de tiempo entre el hecho suscitado y la demanda asistencia por parte de la víctima, a mayor tiempo existe menor probabilidad de identificar las sustancias en toxicología forense, por lo que las sustancias generalmente usadas tiene un metabolismo de vida media corta y se eliminan rápidamente de organismo, convirtiéndose en una limitante para la investigación (2).

El uso premeditado de drogas para manipular la voluntad de las personas ha venido cobrando protagonismo por su asociación con prácticas delictivas de toda índole, con una importante relevancia estadística en agresiones sexuales los últimos años (3). Se define la sumisión química como el uso de una sustancia toxica con fines delictivos de forma que se pueda manipular la voluntad de las personas o modificar su comportamiento, por los efectos farmacológicos de la sustancia administrada evita que la víctima se encuentre en condiciones de prestar su consentimiento legal o de presentar resistencia a su atacante (4).

La estadística mundial según la Organización Mundial de la Salud (OMS) muestra que alrededor de una de cada tres mujeres han sufrido violencia física y/o sexual de pareja o 
violencia sexual por terceros en algún momento de su vida (5), en Ecuador, año 2015 se registraron 15 denuncias por agresión sexual a diario (6).

La falta de estadística y estudios de esta problemática planteó el objetivo de obtener información concisa de la realidad de nuestro entorno con respecto a los delitos tipificados como violación asociados a sumisión química (7). Si analizamos el consumo de las drogas más comunes en nuestro país, encontramos que una de ellas es el alcohol, que ocupaba en el año 2013 el segundo puesto de consumo a nivel de Latinoamérica, y aproximadamente el $5.7 \%$ de la población consume alcohol habitualmente (8). Desde el 10 de agosto del 2014, cuando empezó la aplicación del Código Orgánico Integral Penal (COIP), hasta marzo del 2015, se reportaron 3171 violaciones. Estos datos se encuentran en el último informe de la Fiscalía sobre delitos contra la mujer y el núcleo de la familia (2).

\section{Metodología.}

EL diseño de este estudio fue observacional, analítico y transversal, se realizó en la Unidad de Flagrancia Quito mediante datos de los informes de peritajes medico legales, perteneciente a la Fiscalía de Pichincha y resultados de exámenes toxicológicos en el laboratorio de toxicología del Servicio Nacional de Medicina Legal y Ciencias Forenses, la recolección de datos se efectuó durante todo el año 2017, existieron 197 presuntos casos de violación asociados a presunta sumisión química. Se elaboró una matriz para consolidar la información en un solo cuerpo estadístico y se describió los datos por variable. La codificación de los datos obtenidos se realizó en Microsoft Excel y después de depurar la base de datos, se exportó a un paquete estadístico SPSS versión 24. Las variables cualitativas se describieron empleando frecuencias y porcentajes; en tanto que las variables cuantitativas se analizaron mediante los estadísticos de tendencia central y de dispersión. Se aplicó la prueba de Chi cuadrado y correlación de Pearson para variables cualitativas, obteniendo el grado de significación estadística P y el grado de correlación que existe entre variables a través de la aplicación de V de Cramer y Phi.

\section{Resultados.}

Los datos del estudio se obtuvieron de 68 casos, que están conformados por presuntas víctimas de sexo femenino que acudieron a realizarse un examen médico legal ginecológico en la Unidad de Flagrancia Quito durante el año 2017. De los casos expuestos se obtuvieron muestras biológicas para ejecutar examen toxicológico en el laboratorio de toxicología del Servicio Nacional de Medicina Legal y Ciencias Forenses.

Entre las características demográficas más relevantes encontramos que la edad que prevaleció entre las víctimas fue 17 años, con una edad promedio de 19.5 años con una desviación estándar de \pm 6.7 , una edad mínima de 11 y una máxima de 38 años. 
El $2.9 \%$ correspondieron a ciudadanas extranjeras, el resto correspondió a mujeres ecuatorianas $96.9 \%$ de las cuales el $95 \%$ son oriundas de Pichincha. El estado civil soltero con $85 \%$ es el más frecuente seguido de casado con un $13.2 \%$ y un porcentaje de $1.4 \%$ de divorciadas. El nivel de instrucción fue bachillerato un $44.1 \%$, básico $30.9 \%$ y superior un $25 \%$. El $61.8 \%$ de los casos correspondió a estudiantes, un 13.2\% empleado privado, $8.8 \%$ a personas que realizan trabajo del hogar y empleados públicos, un $4.4 \%$ a empleados privados y un $1.5 \%$ correspondieron a personas desempleadas. Ninguna persona presentó discapacidad.

La agresión sexual bajo el uso de sustancias depresoras del sistema nervioso central no ha sido considerada como un tema de importancia y mucho menos se la ha estudiado en nuestro país, por ello no tenemos datos relevantes en cuanto a esta problemática.

El número de agresores fue una persona en un 54.4\%, dos personas en el $11.8 \%$ de casos y tres y cuatro agresores en un $5.9 \%$.

El lugar de los hechos en un $64.7 \%$ correspondieron a otros, entre los cuales se incluyen principalmente centros de diversión como bares, discotecas, domicilios de amigos, conocidos y personas desconocidas, seguido de un $17.6 \%$ de hechos suscitados en la vía pública, $11.8 \%$ en el hogar de la víctima y el $1.5 \%$ en una institución educativa.

La vía de penetración fue en su mayor porcentaje vaginal en un $19.1 \%$, vía anal y vaginalanal en $4,4 \%$ cada una de ellas. Un aspecto que vale la pena recalcar es que en un $66.2 \%$ de los casos no supieron esclarecer la vía de penetración porque no lo recordaban debido a la amnesia producida por las drogas de sumisión.

Se determinó que el $60 \%$ de las víctimas presentaron lesiones y estas se distribuyen en $36.6 \%$ en lesiones extragenitales que son las distribuidas por toda la anatomía corporal excluyendo el área genital y paragenital, $34.1 \%$ lesiones genitales-extragenitales y $29.3 \%$ lesiones genitales que son las que comprenden en el área vulvar, periné y región anal, aclarando que no se encontraron casos de lesiones paragenitales.

\section{Características de la Sumisión Química.}

Entre las características más importantes encontramos que la hora más frecuente en que se perpetraron los presuntos delitos fueron las 19:00 horas correspondientes al 10.9\% de los casos. Febrero y mayo fueron los meses con mayor presentación con un $16.2 \%$ y $14.2 \%$ respectivamente. Los agresores fueron personas desconocidas en un 57.3\%, personas conocidas en un $33.8 \%$, en las cuales se incluyen relaciones de novio, amigo y esposo. En el $57.3 \%$ de víctimas se realizó el examen y toma de muestras en un lapso mayor a 8 horas. Los resultados de exámenes toxicológicos fueron positivos en un 22\%, negativos el $55.8 \%$ y no realizados un $22 \%$ entre los últimos se incluyen los casos que por haber transcurrido un tiempo mayor a 24 horas desde la hora del suceso, a pesar de haber llegado al 
ISSN: 2602-8085

laboratorio de toxicología no fueron procesadas. En el $26.5 \%$ fueron detectadas algún tipo de drogas y la manera de administración más frecuente fue la voluntaria (ver tabla 1).

Tabla 1. Características de la sumisión química

\begin{tabular}{|c|c|c|c|}
\hline \multicolumn{4}{|c|}{ CARACTERISTICAS DE SUMISIÓN QUIMICA } \\
\hline Parentesco del agreso & & Hora de los hechos & \\
\hline Esposo & $1,40 \%$ & Moda & 19:00 \\
\hline Amigo & $2,90 \%$ & & \\
\hline Novio & $5,80 \%$ & \multirow{2}{*}{ Horas transcurridas desde el hecho } & \\
\hline Conocido & $23,50 \%$ & & \\
\hline \multirow[t]{2}{*}{ Desconocido } & $57,30 \%$ & $<$ a 8 horas & $35,20 \%$ \\
\hline & & $>$ a 8 horas & $57,30 \%$ \\
\hline \multicolumn{4}{|l|}{ Lugar de los hechos } \\
\hline Hogar & $11,70 \%$ & Alcohol y Drogas & \\
\hline Vía pública & $17,60 \%$ & No detectado & $73,50 \%$ \\
\hline Institución educativa & $1,40 \%$ & Alcohol & $22,30 \%$ \\
\hline \multirow[t]{2}{*}{ Otros } & $64,70 \%$ & Benzodiacepinas & $1,40 \%$ \\
\hline & & Cocaína-Marihuana & $1,40 \%$ \\
\hline Mes de los hechos & & Marihuana & $1,40 \%$ \\
\hline Enero & $4,40 \%$ & & \\
\hline Febrero & $16,17 \%$ & Alcoholemia cualitativa & \\
\hline Marzo & $8,80 \%$ & Positivo & $22,00 \%$ \\
\hline Abril & $11,70 \%$ & No detectado & $55,80 \%$ \\
\hline Mayo & $14,70 \%$ & No realizado & $22,00 \%$ \\
\hline Junio & $10,20 \%$ & & \\
\hline Julio & $5,80 \%$ & Tipo de sumisión & \\
\hline Agosto & $10,20 \%$ & Voluntaria & $60,20 \%$ \\
\hline Septiembre & $7,30 \%$ & Involuntaria & $35,20 \%$ \\
\hline Octubre & $4,40 \%$ & & \\
\hline Noviembre & $1,40 \%$ & & \\
\hline Diciembre & $2,90 \%$ & & \\
\hline
\end{tabular}

Fuente: Expedientes de la Unidad de Flagrancia Quito, Servicio Nacional de Medicina Legal Y Ciencias Forenses.

Autor: Md. Jorge Marcelo Quintana Yáne

De los casos estudiados el $26.5 \%$ de los exámenes toxicológicos fueron positivos para algún tipo de droga y de ellos el $83.3 \%$ corresponden a alcohol, 5.6\% benzodiacepinas, $5.6 \%$ marihuana, $5.6 \%$ marihuana-cocaína.

La sumisión química de tipo voluntaria y voluntaria-involuntaria prevaleció con un 60,2\% esta corresponde a que las presuntas víctimas refirieron en su relato antecedentes de consumo de drogas en la mayoría de los casos alcohol por lo que las tomamos en cuenta 
conjuntamente, estas categorías difieren en que en la sumisión voluntaria la víctima indica que estuvo ingiriendo únicamente bebidas alcohólicas en gran cantidad, en la sumisión voluntaria-involuntaria refiere la presunta víctima haber bebido una leve a moderada cantidad de bebidas alcohólicas posterior a lo cual no recuerdan ellos hechos, y el porcentaje minoritario $32.3 \%$ las víctimas no refieren ningún tipo de ingesta alcohólica o de otras drogas.

En base a los datos de alcoholemia cuantitativa se realizó un análisis retrospectivo aplicando la siguiente formula: $\mathrm{Co}=\mathrm{Ct}+\beta \mathrm{t}$

Co = Alcoholemia cuando ocurrió el hecho.

$\mathrm{Ct}=$ Alcoholemia al momento de la extracción. $\mathrm{t}=$ tiempo transcurrido (horas).

$B=$ Tasa de eliminación del alcohol etílico $(0.15 \mathrm{~g} / \mathrm{L} / \mathrm{h})(9)$

Así se obtuvo un valor cuantitativo de alcoholemia en el momento del hecho, y posteriormente este valor fue interpretado cualitativamente, y el estado más prevalente fue estupor con un $35.6 \%$, seguido de estados de embriaguez y ebriedad con un $23.5 \%$, euforia corresponde a un $11.7 \%$ e intoxicación severa a $5.7 \%$.

Se realizó el análisis estadístico Chi cuadrado de Pearson para determinar si existe relación entre las horas transcurridas del hecho y la positividad de alcoholemia. En este caso, se determinó que si existe una relación estadísticamente significativa entre ellas $\left(\mathrm{Chi}^{2} \mathrm{de}\right.$ Pearson $=10.337 \mathrm{p}=0.001$ ), este análisis permite aseverar qué las horas transcurridas desde el hecho hasta la toma de la muestra influyen directamente en el resultado de exámenes toxicológicos y este resultado se contrasta con el tiempo de eliminación del alcohol etílico del cuerpo humano. Además, analizamos el grado de asociación y su direccionalidad usando V de Cramer y Phi. Encontrando que la asociación es moderada e inversa $(\mathrm{V}=0.450 \mathrm{Phi}=-0.450)$, es decir que a mayor número de horas los resultados positivos se reducen considerablemente y viceversa, además indican una relación moderada entre variables.

\section{Discusión}

La sumisión química es un tópico que no ha sido desarrollado ampliamente en investigaciones, existe escasa bibliografía acerca de este tema y los términos empleados alrededor del mundo para referir este problema son muy diversos siendo este el principal obstáculo para realizar revisiones más amplias y obtener una visión clara a nivel mundial, sumado a la dificultades de la víctima de recordar los hechos y la utilización de drogas que son detectadas solo dentro de un corto periodo de tiempo por su breve periodo de vida media. En el presente estudio de investigación se ha procurado utilizar terminología que vaya acorde la mayoría de artículos científicos. Como resultados se obtuvo una población de un promedio de edad de 19.5 años con una edad mínima de 11 años y una máxima de 
38 años, datos que son similares con los resultados de un estudio descriptivo retrospectivo realizado en España por Navarro E. et al. (2), en el cual refiere que la edad de la victimas estuvieron en un rango de edad de 14 a 37 años, también en el estudio realizado por García C. et al. (7), se expresa que el mayor porcentaje de víctimas se encuentran en un rango de edad entre los 15 y 19 años. En el estudio canadiense de Macgregor M. et al. (10), refiere que las víctimas menores de 20 años son más vulnerables a este tipo de delitos concordando con la edad de 17 años que prevaleció en el estudio realizado.

El estado civil soltero fue el más relevante con un $85.2 \%$, seguido del estado civil casado y en un mínimo porcentaje divorciado no se encontró estudios específicos de violación y sumisión química que describan características demográficas como la descrita, ya que estos están enfocados a la recolección de variables específicas de sumisión química y no a la demografía de la población, sin embargo, en el estudio De la Garza J. et al. (11), enfocado a violación sexual expone que el $67.7 \%$ de las víctimas eran solteras un $26.6 \%$ eran casadas y $5.7 \%$ no tenían pareja, en esta última categoría incluyen a divorciadas, separadas, viudas y madres solteras, datos muy parecidos también a los resultados del estudio de De la Cruz M.et al. (12), que indican un $79 \%$ de solteras $13 \%$ de casadas y $8 \%$ de separadas o divorciadas siendo estos datos concordantes con los resultados obtenidos.

El nivel de instrucción en el estudio fue bachillerato el prevalente con un $44.1 \%$, seguido de básico correspondiente al 30.8\% y un menor porcentaje cursó instrucción superior y pertenece al 25\%, en el estudio de De la Garza J. et al. (11), describe que 5.2\% tenían nivel de instrucción básica $83.2 \%$ bachillerato y el $7.9 \%$ de la población estudiada contaban con educación superior, según el estudio De la Cruz M.et al. (12), el 42.9\% de las victimas contaban con instrucción superior, $29.9 \%$ tendría estudios secundarios correspondiente a bachillerato y educación básica en un porcentaje de $27.3 \%$, por lo que no existe concordancia entre los resultados pero probablemente es debido a la ubicación geográfica de la muestra estudiada ya que en los dos primeros estudios corresponden a américa latina mientras que el segundo corresponde a poblaciones europeas donde estadísticamente el nivel educativo es mayor.

La ocupación que prevaleció fue estudiantes con el $61.7 \%$ seguido de empleados privados y trabajo del hogar en un porcentaje de $8.8 \%$ cada uno de ellos, todos estos datos están relacionados con la edad de las presuntas víctimas y además es el grupo etario en el cual el consumo de alcohol y drogas ha ido en aumento según datos de estadísticas nacionales. En el estudio no se encontró ninguna persona con discapacidad que está en relación con el resultado del estudio de Xifró A. et al. (13), en el cual en una muestra de 114 víctimas se presentaron un solo caso de discapacidad física y dos casos de discapacidad intelectual.

Las lesiones genitales se presentaron en un total de $60 \%$ de los casos contrastando con los datos obtenidos por Larsen M. et al (14) lesiones anogenitales en $26.8 \%$ dato que es mucho menor al mencionado. 
En lo que respecta a las variables analizadas en el contexto propio de sumisión química los resultados fueron similares al de este estudio con discrepancias y algunas diferencias en ciertos aspectos; estudios de Navarro, Isorna, Xifró, García-Caballero, Torres son los más representativos; es así que el parentesco del agresor con la víctima en el estudio fueron personas desconocidas en el $57.33 \%$ y conocidas en el $33.8 \%$ en este último se incluyen relaciones de amigo, esposo y novio, Navarro E. et al. (2), expresa en sus resultados que el porcentaje de personas conocidas fue un $47.4 \%$ de los casos y en el $84 \%$ de ellas no los une ningún parentesco, concordando que existe una mayor proporción de personas desconocidas que perpetran este tipo de delitos, en la revisión bibliográfica de Isorna refiere que en un estudio realizado en España predominaron los agresores conocidos en un porcentaje del 47\% , así también en el estudio de Xifró A. et al. (13), el porcentaje de agresores conocidos fue un $33.7 \%$, desconocidos un $29.1 \%$, y amigo, familiar o pareja en un $32.7 \%$, en la variable analizada es discordante en los dos últimos estudios y esta no se debe a la situación geográfica como encontramos en una variable anteriormente analizada.

Xifró A. et al. (13), en su estudio únicamente menciona como lugar de los presuntos hechos a sitios privados correspondientes al 55.4\% de la población estudiada, en el estudio de Navarro E. et al. (2), se manifiesta que casi la mitad de los casos $47.4 \%$ ocurren en el propio domicilio de la víctima, Larsen et al. (14), estudia una población considerable pero toma en cuenta todos los casos de violación, no es exclusivo de sumisión química y refiere que la mayoría de casos ocurren en el domicilio y en menor porcentaje en sitios diferentes al mencionado, en el estudio realizado obtuvimos que la categoría otros representada por el $64.7 \%$ de los casos, la cual integra bares, discotecas, domicilios de amigos, conocidos y personas desconocidas esta categoría fue la más relevante, mientras que en un porcentaje de solo el $11.8 \%$ los hechos fueron perpetrados en el domicilio de la presunta víctima.

Con relación al mes de los hechos se obtuvo que en los meses de febrero y mayo se obtuvieron mayor presentación de casos, con un $16.17 \%$ y $14.7 \%$ respectivamente, esto puede deberse a que en nuestro país tenemos dos fechas de asueto en esos meses y especialmente en febrero es un feriado extenso en el cual se aumenta notoriamente la ingesta alcohólica Navarro E. et al. (2), en su estudio refiere que mayo y diciembre son los meses en que más se presentaron casos con un $16 \%$ cada uno de ellos, mientras que en marzo y octubre no se identificaron casos, datos que no están concordantes con los meses de menor incidencia identificados en nuestro estudio se da en los meses de noviembre y diciembre con un $1.4 \%$ y $2.9 \%$ respectivamente y probablemente podría deberse a que estas fechas son más de unión familiar en nuestro país.

La hora de los hechos en que más sucesos se presentaron fueron las 19:00, en esta variable tenemos una marcada diferencia ya que en el estudio de Xifró A. et al. (13), los hechos suceden en un intervalo de las 00:00 a 07:59 horas y en un horario más general nos presenta el estudio de Torres P. et al. Que refiere que el $56.67 \%$ de los delitos ocurrieron en horario nocturno y en el fin de semana. 
En el estudio recolectamos información de carácter categórico cualitativo en lo que respecta a las horas transcurridas desde el hecho hasta la valoración y toma de muestras siendo los resultados que en el $35.2 \%$ de las víctimas se obtuvo las muestras antes de las ocho horas mientras que en el $57.3 \%$ se realizó dicho procedimiento posterior a este tiempo. Xifró A. et al. (13), hace referencia a dos categorías 12 horas o menos con un porcentaje de $59.6 \%$ del total de casos y 24 horas o menos con un $79.8 \%$ lo cual responde a que la mayor parte de los casos han sido tomadas las muestras hasta un lapso de 24 horas y no nos da el corte necesario para poder realizar una comparación adecuada; al igual que el estudio de Navarro E. et al. (2), que nos presenta el rango de horas que fue de un mínimo de 2 horas y un máximo de 51 horas, con un tiempo medio de 18.26 horas.

Se detectó en los exámenes toxicológicos de este estudio alcohol en el $22.3 \%$, benzodiazepinas, marihuana y una mezcla de cocaína y marihuana en un porcentaje de $1.4 \%$ cada una. Navarro E. et al. (2), encontró básicamente drogas idénticas a las del estudio presentado, aunque en porcentajes distintos así en mayor proporción se encontró cocaína $26.3 \%$, cannabinoides $15.8 \%$, benzodiacepinas $15.8 \%$, alcohol $10.5 \%$ y anfetaminas 5.3\%. García C. et al. (7), en su estudio expresa que la droga más prevalente es el etanol correspondiente al $61.7 \%$ de los casos, psicofármacos en el $40.2 \%$ y drogas ilícitas en el $27.1 \%$ siendo estos resultados muy concordantes con los obtenidos, según García R. et al. (4), el alcohol etílico se presentó en $57.5 \%$ de los casos recibidos y es la sustancia más frecuentemente detectada, las benzodiacepinas fueron detectadas en un $16.45 \%$ de los casos y en mucha menor frecuencia se encontraron cocaína y derivados cannábicos.

La manera de sumisión química no se la menciona en los estudios citados sin embargo refieren que un gran número de agresiones sexuales asociadas a sumisión química están asociadas con el consumo previo de alcohol ya sea en escasa o gran cantidad, y lo describen como un factor de riesgo muy importante en las agresiones sexuales. Así en este estudio encontramos que en el $60.2 \%$ de la población según el relato estuvieron consumiendo bebidas alcohólicas las mismas que se las clasificó en voluntarias; cuando consumieron una escasa cantidad se las catalogó como de tipo voluntario-involuntario.

En cuanto a la relación realizada de resultados positivos de alcohol y el tiempo transcurrido en el estudio se obtuvo una relación estadísticamente significativa $(p=0.001)$ que expresa que las horas de toma de muestra optimas deben ser menor a ocho horas, esta información está conexa con el tiempo de eliminación del alcohol, García C. et al. (4), refiere en su estudio que la toma de muestras dentro de las 6 horas nos da resultados positivos en el $100 \%$ de los casos, de 6 a 12 horas el $90.6 \%$ son positivos, de 13 a 14 horas $40 \%$ de positividad, en muestras tomadas mayor a 36 horas no existió ningún resultado positivo. 


\section{Conclusiones}

- El tiempo que transcurre desde el hecho hasta la toma de muestra se relaciona de manera directa con los resultados de exámenes toxicológicos; teniendo un mayor porcentaje de positividad en los resultados de muestras que se obtuvieron en las primeras ocho horas posteriores al hecho, por lo tanto, validamos la hipótesis de trabajo y rechazamos la hipótesis nula.

- Las presuntas víctimas tuvieron una edad entre 11 y 38 años, el estado civil soltero prevaleció, el grado de instrucción fue bachillerato y la mayor parte de ellas fueron estudiantes en el contexto de características demográficas.

- Las características de la agresión sexual en la esfera de sumisión química fueron similares a las encontradas en bibliografía que estudian la violación como tal, teniendo como datos relevantes que en la mayoría de casos fue un solo agresor, desconocido para la víctima, las lesiones más prevalentes fueron extragenitales.

- La droga más utilizada en el Ecuador para el cometimiento de delitos en contra la libertad sexual fue el alcohol, su manera de administración fue voluntaria en la mayor parte de los casos, y se pudo determinar que en un gran porcentaje de los delitos perpetrados el estado de conciencia estupor, por lo tanto, utilizando el examen retrospectivo y la clasificación en una escala cualitativa se puede contribuir notablemente en un proceso judicial aportando una prueba objetiva y científica que refleja el estado de conciencia de la víctima al momento del hecho, de ahí radica la importancia de realizar una toma de muestra oportuna y correcta.

\section{Referencias Bibliográficas}

Du Mont J, Mc Donals S, Rotbard N, Smith N. Drug-facilitated sexual assault in Ontario, Canada: Toxicological and DNA findings. Journal of forensic and legal Medicine. 2010; 17.

Navarro E, Vega C. Agresiones sexuales facilitadas por sustancias psicoactivas, detectadas en el instituto de medicina legal de Alicante en el cuatrienio 2009 - 2012. Gac int.cienc.forense. 2013.

Santurtun A. Dificultades forenses y procesales de la sumisión quimica. Revista Española de Medicina Legal. 2017.

Garcia R, Soria M. Sumisión química: reto para el toxicólogo forense. Revista Española de Medicina Lega. 2011.

Organización Mundial de la Salud. Violencia de pareja y violencia sexual contra la mujer. 2016. 
Espinoza M, Ortiz S. 15 violaciones se denuncian a diario en el país. El Comercio. 2015.

Garcia C. Estudio de prevalencia en casos de presuntos delitos contra la libertad sexual analizados en el Instituto Nacional de Toxicología y Ciencias Forenses. 2015.

Instituto Ecuatoriano de Estadísticas y Censos (INEC). Consumo de Alcohol..

Ferrari L. Análisis toxicológico de etanol y su interpretación forense. Ciencia Forense Latinoamericana. $2008 ; 2$.

McGregor M, Ericksen J, Ronald L. Rising incidence of hospital reported drug-facilitated sexual assault in a large urban community in Canada. Retrospective population-based study. Public Health. 2004; 95.

De la Garza J. lementos para el estudio de la violación sexual. Salud Pública de México. 1997; 33.

De la Cruz M. Estudio descriptivo de una muestra de víctimas de agresión sexual. Psicopatología Clínica, Legal y Forense. 2014.

Xifró A. Estudio prospectivo de la sumisión química con finalidad sexual en Barcelona. Medicina Clinica Elsevier. 2015.

Larsen M. Sexual assault: a descriptive study of 2500 female victims over a 10-year period. Royal College of Obstetricians and Gynaecologists. 2014. 


\section{Para citar el artículo indexado.}

Quintana J., Galarza M., Hinojosa A. \& Cáceres V.. (2019) Sumición química en presuntas víctimas de violencia sexual. Revista electrónica Ciencia Digital 3(1.1), 216227.

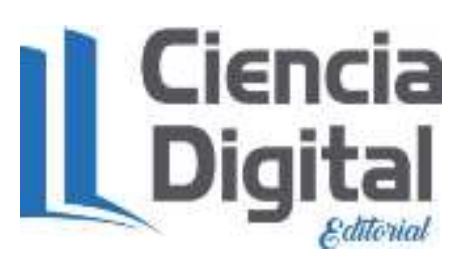

El artículo que se publica es de exclusiva responsabilidad de los autores y no necesariamente reflejan el pensamiento de la Revista Ciencia Digital.

El articulo queda en propiedad de la revista y, por tanto, su publicación parcial y/o total en otro medio tiene que ser autorizado por el director de la Revista Ciencia Digital.
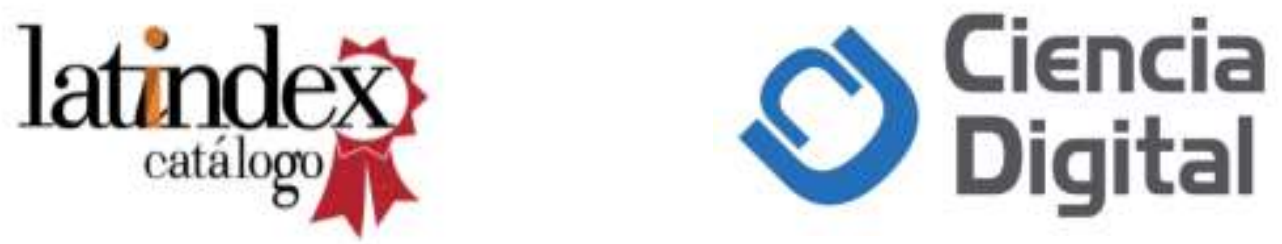